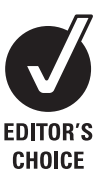

CHOICE

\title{
Obesity and the risk of psoriatic arthritis: a population-based study
}

\author{
Thorvardur Jon Love, ${ }^{1}$ Yanyan Zhu, ${ }^{2}$ Yuqing Zhang, ${ }^{2}$ Lindsay Wall-Burns, ${ }^{3,4}$ \\ Alexis Ogdie, ${ }^{5}$ Joel M Gelfand, ${ }^{6}$ Hyon $\mathrm{K} \mathrm{Choi}^{7}$
}

'Department of Science,

Education, and Innovation,

Landspitali University Hospital,

Fossvogur, Reykjavik, Iceland

${ }^{2}$ The Clinical Epidemiology Unit, Department of Medicine Boston

University School of Medicine,

Boston, Massachusetts, USA

${ }^{3}$ Arthritis Research Centre of

Canada, Vancouver, Canada

${ }^{4}$ Department of Dermatology

and Skin Science, University of

British Columbia, Vancouver,

Canada

${ }^{5}$ Department of Medicine/

Rheumatology, University of

Pennsylvania, Philadelphia,

Pennsylvania, USA

${ }^{6}$ Department of Dermatology,

University of Pennsylvania,

Philadelphia, Pennsylvania, USA

${ }^{7}$ Section of Rheumatology

and the Clinical Epidemiology

Unit, Boston University

School of Medicine, Boston,

Massachusetts, USA

\section{Correspondence to}

Hyon K Choi, Section of Rheumatology and the Clinical Epidemiology Unit, Boston University School of Medicine, 650 Albany Street, Suite 200, Boston, MA 02118, USA

hchoius@bu.edu

Received 9 February 2012 Accepted 26 February 2012

\section{ABSTRACT \\ Background Obesity is associated with an increased} risk of psoriasis; however, its potential impact on the risk of psoriatic arthritis (PsA) remains unclear.

Objectives To evaluate the association between body mass index (BMI) and the risk of PsA among patients with psoriasis from the general population.

Methods The authors conducted a cohort study using data from The Health Improvement Network, an electronic medical records database representative of the UK general population, collected between 1995 and 2010. The exposure of interest was the first BMI measured after psoriasis diagnosis and endpoints were incident cases of physician-diagnosed PsA. The authors estimated the RR of PsA after adjusting for age, sex, and histories of trauma, smoking and alcohol consumption. Results Among 75395 individuals with psoriasis (43\% male, mean follow-up of 5 years, and mean age of 52 years), 976 developed PsA (incidence rate, 26.5 per 10000 person-years). The PsA incidence rates increased with increasing BMI. Compared with psoriasis patients with $\mathrm{BMI}<25 \mathrm{~kg} / \mathrm{m}^{2}$, the RRs for developing PsA were 1.09 (0.93-1.28) for BMls from 25.0 to 29.9, 1.22 (1.02-1.47) for BMls from 30.0 to 34.9 and 1.48 (1.20-1.81) for BMls $\geq 35.0$. In our secondary analysis among all individuals, regardless of psoriasis ( $\sim 2$ million), the corresponding multivariate RRs tended to be stronger $(1.0,1.17,1.57,1.96$; $p$ for trend <0.001).

Conclusions This general population study suggests that obesity is associated with an increased risk of incident PsA and supports the importance of weight reduction among psoriasis patients who often suffer from the metabolic syndrome and obesity.

\section{INTRODUCTION}

Psoriatic arthritis (PsA) is a progressive and often destructive inflammatory joint disease associated with psoriasis. ${ }^{1-3}$ The inflammatory arthritis of PsA adds substantially to the disease burden for psoriasis patients, with frequent joint destruction associated with cartilage erosions, bone damage and joint fusion. The negative impact of psoriasis may not be limited to its cutaneous or psychosocial manifestations, ${ }^{4}$ with emerging evidence indicating that psoriasis is associated with the metabolic syndrome ${ }^{5}$ and may lead to myocardial infarction, ${ }^{7}$ hypertension and diabetes. ${ }^{8}$ Despite these lifelong burdens of PsA, its causes and risk factors are poorly understood with a lack of large-scale data.
PsA is common in patients with psoriasis, affecting about $6-10 \%$ of psoriasis patients overall and substantially more patients with more extensive skin disease (up to $40 \%$ ). ${ }^{1-3}$ In most patients with PsA, the symptoms do not develop until years after the onset of cutaneous psoriasis. As a result, patients with psoriasis represent a unique opportunity to study individuals at very high risk of developing a chronic inflammatory arthropathy (ie, PsA). ${ }^{1}$ To determine which patients with psoriasis are at the greatest risk of developing PsA, it is essential that risk factors be identified using robust epidemiological approaches. ${ }^{1}$

The adipose tissue in obese individuals is a site of overproduction of important inflammatory cytokines such as tumour necrosis factor $\alpha$, interleukin 1 , IL- 6 and IL-8, ${ }^{910}$ and the resulting abnormal inflammatory milieu may contribute to the pathophysiology of psoriatic conditions. ${ }^{10}$ Obesity has been found to be associated with an increased risk of psoriasis, ${ }^{11}$ but its link to the risk of PsA remains less clear. Recent cross-sectional studies have found that PsA patients have a higher body mass index (BMI) than healthy controls, ${ }^{12} 13$ and patients with PsA have a $61 \%$ higher odds of having obesity than psoriasis patients. ${ }^{13}$ A case series study ( $\mathrm{n}=197$ for PsA) reported that recalled BMI at 18 years of age was associated with an increased risk of PsA, whereas current BMI was not. ${ }^{14}$

In this study, we examined the relation between BMI and the risk of incident PsA among 75395 individuals with psoriasis from the general population.

\section{METHODS}

Study population

The Health Improvement Network (THIN) is a computerised medical record database entered by general practitioners (GP) in the UK. Data on approximately 7.3 million patients are systematically recorded and sent anonymously to THIN. Because the National Health Service in the UK requires every individual to be registered with a GP regardless of health status, THIN is a populationbased cohort representative of the UK general population. The computerised information includes demographics, details from GPs' visits, diagnoses from specialists' referrals and hospital admissions, results of laboratory tests, and additional health information recorded systematically including height, weight, blood pressure, smoking and vaccinations. The Read classification is used to code specific diagnoses, and a drug dictionary based on data 
from the Multilex classification is used to code drugs. ${ }^{12} 13$ Health information is recorded on site at each practice using a computerised system with quality control procedures to maintain high data completion rates and accuracy. Thus, THIN data represent routine medical practice in a population-based setting. ${ }^{15}$

Our primary study population consisted of psoriasis patients aged 20-89 years and enrolled in THIN at any time between January 1995 and May 2010 ( $n=75$ 395). Our secondary study populations included the cohort of individuals with or without psoriasis at baseline ( $\mathrm{N}=2026139)$ and that limited to patients with incident psoriasis $(n=27980)$. Study cohort members had to be free of PsA before entering the study and were required to have two or more years of enrolment with the GP to help ensure exclusion of prevalent PsA. An individual's start (index) date corresponded to the date of the first recorded BMI after all these eligibility criteria were met. Members of this cohort were followed until the date of one of the following endpoints: PsA diagnosis, 90th birthday, death or end of study period, whichever came first.

\section{Ascertainment of psoriasis and PsA}

Validity of THIN Read codes for psoriasis has been documented based on a sample of 4900 psoriasis patients. ${ }^{16}$ With a $95 \%$ response rate in a GP survey for clinical confirmation of psoriasis, the Read codes for psoriasis in THIN showed a positive predictive value (PPV) of $90 \%$ of clinical confirmation. Therefore, we defined psoriasis based on its Read code in THIN.

Although validity of PsA Read codes has not been assessed in THIN, the positive predictive value (PPV) of a single code for PsA has been reported to be over $90 \%$ in another electronic medical record database. ${ }^{17}$ The use of diagnostic codes plus disease-modifying antirheumatic drug data definitions only slightly and significantly improved the specificity for each diagnosis, but at the cost of a dramatic reduction in sensitivity. ${ }^{17}$ Therefore, we defined PsA based on its Read diagnostic code in this study.

\section{Assessment of exposure and covariates}

Demographics, lifestyle factors and history of trauma were collected from the database before the index date. THIN lifestyle exposure variables have been collected prospectively and successfully used in previous analyses by confirming anticipated associations such as those among alcohol, BMI and gout; ${ }^{18}$ smoking and lung cancer; ${ }^{19}$ smoking, obesity and myocardial infarction; ${ }^{20}$ and alcohol and paroxysmal atrial fibrillation. ${ }^{21}$ Our exposure of interest was BMI, defined as the patient's body mass in kilograms divided by the square of his or her height in metres. We analysed the impact of the first recorded BMI after all these eligibility criteria were met (eg, the first BMI recorded after psoriasis diagnosis in our primary study cohort). We repeated our analysis using updated BMI as a time-varying exposure. History of trauma was included as a covariate as well, as this has also been reported to be associated with PsA. ${ }^{22} 23$

\section{Statistical analysis}

We calculated the eligible person-time of follow-up and incidence rates according to four BMI categories: <25, 25-29.9, $30-34.9$ and $\geq 35 \mathrm{~kg} / \mathrm{m}^{2}$, corresponding to the WHO's classification of normal weight, overweight, obese class I and obese class II or III, respectively. ${ }^{24}$ We used a Cox proportional hazards model to compute the multivariate RR for incident PsA adjusting for sex, age, smoking status (never/past/current), alcohol use (six categories) and history of trauma.
We repeated our analysis in the THIN population by additionally including those without psoriasis status ( $n=1950744)$. In this analysis, we examined the impact of including the diagnosis of psoriasis in the model, as BMI is a known risk factor for psoriasis and, thus, can act as a potential causal intermediate. We also repeated the analysis limited to patients with incident psoriasis $(n=27$ 980). This allowed us to examine the potential impact of psoriasis duration on varying risks of PsA. For all RRs, we calculated $95 \%$ CIs. All p values are two-sided. All statistical analyses were performed using SAS software, V.9.2 (SAS Institute, Cary, North Carolina, USA).

\section{RESULTS}

Our primary analysis included 75395 psoriasis patients for a combined follow-up time of 368302 person-years, during which 976 cases of incident PsA were diagnosed. The incidence rate of PsA in this population was 26.5 per 10000 person-years. Table 1 summarises the baseline characteristics of the study cohort according to BMI.

There were fewer men overall, which was most notable in the highest and lowest BMI categories. Lower BMI categories tended to have more current smokers and fewer past smokers. Other variables were similarly distributed across BMI categories.

Higher categories of BMI were associated with an increased incidence of PsA ( $p$ for trend <0.001) (table 2 and figure 1). Compared with psoriasis patients with BMIs $<25$, those with BMIs of 25-29.9, 30.0-34.9 and $\geq 35.0$ had age- and sex-adjusted RRs of 1.1, 1.24 and 1.52, respectively ( $p$ for trend $<0.001$ ). After adjusting for age, sex, smoking, alcohol consumption and history of trauma, these RRs were slightly attenuated but remained significant ( $p$ for trend $<0.001$ ) (table 2). When we repeated our analysis using updated BMI as a time-varying exposure, our results did not change materially ( $p$ for trend $<0.001$ ).

When we repeated the analysis by additionally including those without psoriasis status ( $\mathrm{n}=1950744)$, higher categories of BMI were associated with an increased risk of PsA ( $p$ for trend <0.001) (table 3 and figure 2). Compared with individuals with BMIs $<25$, those with BMIs of 25-29.9, 30.0-34.9 and $\geq 35.0$ had age- and sex-adjusted RRs of $1.17,1.60$ and

Table 1 Baseline characteristics according to body mass index for 75395 patients with psoriasis

\begin{tabular}{|c|c|c|c|c|c|}
\hline & \multicolumn{4}{|c|}{ Body mass index $\left(\mathrm{kg} / \mathrm{m}^{2}\right)$} & \multirow[b]{2}{*}{$\geq 35.0$} \\
\hline & All & $<25.0$ & $25.0-29.9$ & $30.0-34.9$ & \\
\hline $\mathrm{N}$ & 75395 & 26263 & 27147 & 14088 & 7897 \\
\hline Age (mean, years) & 52.2 & 50.0 & 54.2 & 53.5 & 50.1 \\
\hline Male & 43.4 & 35.0 & 51.6 & 48.3 & 34.8 \\
\hline \multicolumn{6}{|l|}{ Smoking status } \\
\hline Never & 43.4 & 42.5 & 43.8 & 43.1 & 45.7 \\
\hline Previous & 26.0 & 20.3 & 28.2 & 31.1 & 28.6 \\
\hline Current & 28.8 & 35.6 & 26.4 & 23.7 & 23.4 \\
\hline Missing & 1.79 & 1.66 & 1.64 & 2.04 & 2.27 \\
\hline $\begin{array}{l}\text { Alcohol intake, no. } \\
\text { of servings per } \\
\text { week (mean) }\end{array}$ & 7.85 & 7.60 & 8.55 & 7.88 & 6.08 \\
\hline 0 & 25.3 & 24.2 & 23.6 & 27.0 & 32.0 \\
\hline $1-9$ & 32.1 & 33.8 & 32.1 & 30.5 & 29.3 \\
\hline $10-24$ & 17.1 & 16.4 & 19.6 & 16.7 & 11.8 \\
\hline $25-41$ & 4.03 & 3.32 & 4.86 & 4.41 & 2.87 \\
\hline$>41$ & 1.99 & 1.94 & 2.04 & 2.15 & 1.72 \\
\hline Missing & 19.5 & 20.3 & 17.9 & 19.3 & 22.4 \\
\hline Trauma & 41.6 & 40.2 & 42.1 & 42.5 & 42.9 \\
\hline
\end{tabular}

Values are percentages unless otherwise noted. 
Table 2 RR of incident psoriatic arthritis by body mass index among patients with psoriasis

\begin{tabular}{|c|c|c|c|c|c|c|c|c|c|}
\hline & \multicolumn{8}{|c|}{ Body mass index $\left(\mathrm{kg} / \mathrm{m}^{2}\right)$} & \multirow[b]{2}{*}{ p For trend } \\
\hline & $<25.0$ & & $25.0-29$. & & $30.0-34$. & & $\geq 35.0$ & & \\
\hline $\begin{array}{l}\text { Cases of psoriatic } \\
\text { arthritis, N }\end{array}$ & 300 & & 341 & & 197 & & 138 & & - \\
\hline $\begin{array}{l}\text { Incidence rate/10 } 000 \\
\text { person-years }\end{array}$ & 23.1 & $(20.6-25.9)$ & 25.4 & $(22.8-28.2)$ & 29.3 & $(25.4-33.7)$ & 38.0 & $(32.0-44.9)$ & $<0.001$ \\
\hline $\begin{array}{l}\text { Age- and sex-adjusted } \\
\text { RR ( } 95 \% \mathrm{CI})\end{array}$ & 1.00 & (reference) & 1.10 & $(0.94-1.29)$ & 1.24 & $(1.03-1.49)$ & 1.52 & $(1.24-1.86)$ & $<0.001$ \\
\hline $\begin{array}{l}\text { Multivariate RR } \\
(95 \% \mathrm{Cl})^{*}\end{array}$ & 1.00 & (reference) & 1.09 & $(0.93-1.28)$ & 1.22 & $(1.02-1.47)$ & 1.48 & $(1.20-1.81)$ & $<0.001$ \\
\hline
\end{tabular}

*The multivariate adjusted model includes age, sex, smoking status, alcohol consumption and history of trauma.

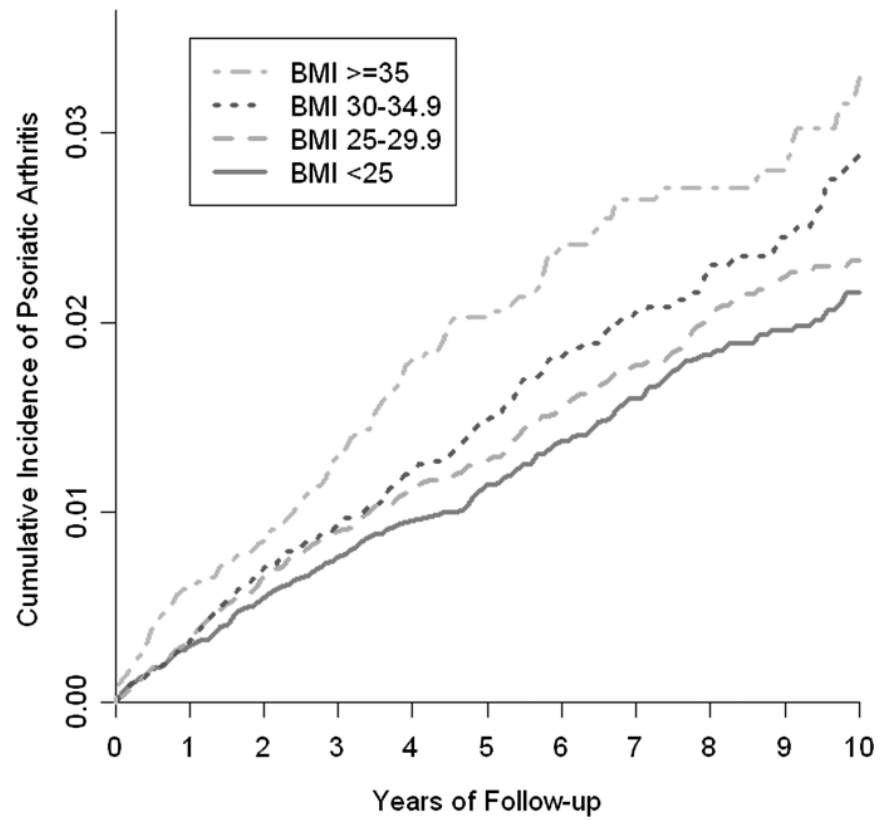

Figure 1 Time to psoriatic arthritis according to body mass index (BMI) category among patients with psoriasis $(\mathrm{N}=75395)$.

2.01, respectively, ( $p$ for trend $<0.001$ ), which were larger than those in our primary analyses. Further adjustment for the history of psoriasis in this secondary analysis modestly attenuated the association $(1.0,1.12,1.46,1.75 ; \mathrm{p}$ for trend $<0.001)$. When we limited our analysis to 27980 patients with incident psoriasis, the RR for the highest BMI category was 1.65 ( $p$ for trend, 0.005).

\section{DISCUSSION}

Our primary objective was to evaluate the association between $\mathrm{BMI}$ and the risk of PsA among patients with psoriasis from the general population. We found that increasing BMI among patients with psoriasis was associated with an increasing risk of incident PsA, demonstrating a dose-response relation. When we examined the risk in all eligible individuals regardless of psoriasis status, the magnitude of association became larger. These associations were independent of other potential confounders such as age, sex, trauma, smoking and alcohol intake. These data provide the first general population-based evidence that obesity is associated with an increased risk of developing PsA among psoriasis patients as well as in the general population.

A recent cross-sectional study reported that PsA patients have a higher BMI than psoriasis patients. ${ }^{13}$ Another study based on psoriasis patients recruited consecutively from the dermatology clinic of the University of Utah reported an independent association with recalled BMI at age 18, but no such associations with current BMI among psoriasis patients. ${ }^{14}$ However, as the current BMI data in that study were measured after the study recruitment when PsA status was determined, they reflect adiposity levels after the onset of PsA (as opposed to BMI before the onset of PsA). Furthermore, because the recalled BMI data at age 18 do not take into account BMI changes that occurred from age 18 leading up to and following the development and diagnosis of psoriasis, its utility for predicting the future risk of PsA based on adiposity status of psoriasis patients is unclear. Our results filled this knowledge gap by examining the relation between BMI after psoriasis diagnosis (and before PsA onset) and the risk of PsA.

Potential explanations for this increased risk for PsA among obese psoriasis patients include the inflammatory load associated with adiposity, contributing further to that associated with their underlying psoriatic condition. Obesity is associated with an overproduction of inflammatory cytokines including IL-1, IL-6, IL-8 and tumour necrosis factor $\alpha$, and there is a correlation between the level of adiposity and the measured peripheral levels of associated inflammatory cytokines. ${ }^{9} 10$ Randomised trial data showing beneficial additive effects of weight $\operatorname{loss}^{25}$ and case reports of psoriasis clearing with reducing obesity ${ }^{26-28}$ suggest that this inflammatory milieu provided by the overabundance of adipose tissue may be important in the pathogenesis of psoriatic conditions. An alternative speculation for the increased risk observed here could be that mechanical wear, secondary to obesity, could trigger PsA in load-bearing joints, with subsequent spread of the inflammatory arthritis to other joints.

Our findings may have practical implications for the management of psoriasis patients, as PsA is common in these patients, affecting about $6-10 \%$ of psoriasis patients overall and substantially more patients with extensive skin disease (up to $40 \%$ ). ${ }^{1-3}$ Thus, a modifiable risk factor, such as obesity, requires a relatively small number needed to treat among psoriasis patients compared with the general population context with a lower background risk of developing PsA. Although a randomised trial would be needed to definitively establish the usefulness of weight reduction in reducing the risk of PsA, this type of study may be difficult to perform, given the large number of psoriasis patients and trial durations necessary for sufficient cases of PsA to occur, the high costs and anticipated ethical issues. Meanwhile, as weight reduction in obese individuals has multiple health benefits, including reduced cardiovascular and mortality risks, these benefits appear all the more relevant for obese psoriasis patients to help prevent 
Table 3 RR for incident psoriatic arthritis by body mass index in the general population

\begin{tabular}{|c|c|c|c|c|c|c|c|c|c|}
\hline & & & & Body mass ir & idex $(k$ & $\left./ \mathrm{m}^{2}\right)$ & & & \\
\hline & $<25.0$ & & 25.0 & 29.9 & 30.0 & 34.9 & $\geq 35.0$ & & p For trend \\
\hline Cases of psoriatic arthritis, $\mathrm{N}$ & 551 & & 591 & & 363 & & 231 & & - \\
\hline Incidence rate/10 000 person-years & 1.23 & $(1.13-1.34)$ & 1.47 & $(1.35-1.59)$ & 2.05 & $(1.84-2.27)$ & 2.67 & $(2.34-3.04)$ & $<0.001$ \\
\hline Age- and sex-adjusted RR (95\% Cl) & 1.00 & (reference) & 1.17 & $(1.04-1.32)$ & 1.60 & $(1.40-1.83)$ & 2.01 & $(1.72-2.34)$ & $<0.001$ \\
\hline Multivariate RR $(95 \% \mathrm{Cl})^{*}$ & 1.00 & (reference) & 1.17 & $(1.04-1.31)$ & 1.57 & $(1.38-1.80)$ & 1.96 & $(1.68-2.29)$ & $<0.001$ \\
\hline
\end{tabular}

*The multivariate adjusted model includes age, sex, smoking status, alcohol consumption and history of trauma.

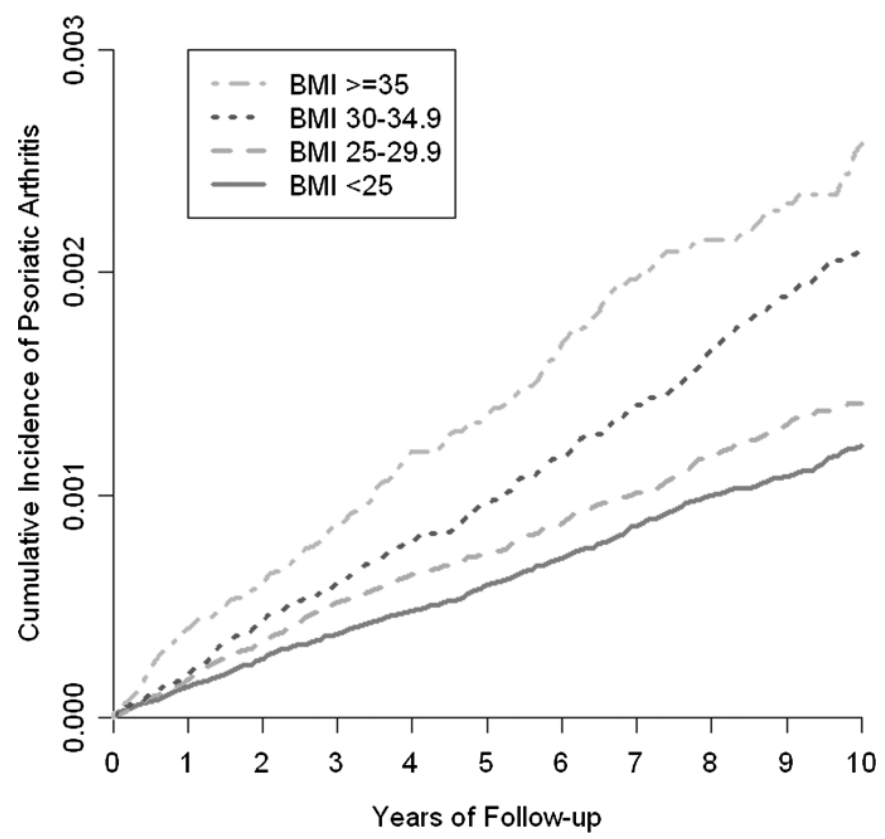

Figure 2 Time to psoriatic arthritis according to body mass index (BMI) category in all eligible adults $(\mathrm{N}=2026139)$ with or without psoriasis at baseline.

their excess risk of PsA along with co-morbidity and mortality complications.

The strengths and limitations of our study deserve comment. This study was performed using a large UK general practice database; therefore, findings are likely to be applicable to the general population. While the designs of previous studies on the topic made it difficult to establish the temporal relations between BMI and the risk of PsA, our study design, including the time-point of exposure measurement and the incident case outcome analysis, enabled us to establish temporal relations between these factors. ${ }^{13} 14$ Uncertainty surrounding diagnostic accuracy is a potential concern in studies that identify cases from administrative databases. However, our databases are actually electronic medical records used for patients' care, thus the overall accuracy is expected to be higher, as reflected in many validation studies of important outcomes. ${ }^{1629-33}$ As is well reflected in the high PPV estimates for a psoriasis code in THIN $(90 \%),{ }^{16}$ and for a PsA code in another electronic medical record database $(>90 \%),{ }^{17}$ our cohort definition (psoriasis patients) and outcome (PsA) belong to this group of 'specific' diagnoses, likely due to the characteristic skin lesions of psoriasis and the clinical context in which PsA is diagnosed. PsA is among those specific diagnoses that are usually first made or confirmed by rheumatologists (unlike rheumatoid arthritis (RA)). ${ }^{17}$ This is likely because both referring physicians and patients are not as familiar with this diagnosis as they are with other common types of arthritis (eg, RA or osteoarthritis) and there are no widely available blood tests that flag the diagnosis of PsA (eg, rheumatoid factor or anticyclic citrullinated protein for RA).

In conclusion, this general population study suggests that obesity is associated with an increased risk of incident PsA and supports the importance of weight reduction among psoriasis patients who often suffer from the metabolic syndrome and obesity.

Contributors All authors participated in the conception, design and analyses of the study. TJL and HKC drafted the manuscript and are guarantors. All authors contributed to interpretation of the results.

Funding This work was supported in part by grants from the NIAMS (P60AR047785) and Boston University School of Medicine. The funding sources had no role in the design, conduct or reporting of the study, or in the decision to submit the manuscript for publication.

Competing interests None.

Ethical approval The current study was approved by the NHS Research Ethics Committee (09/H0305/75).

Provenance and peer review Not commissioned; externally peer reviewed.

\section{REFERENCES}

1. Ogdie A, Gelfand JM. Identification of risk factors for psoriatic arthritis: scientific opportunity meets clinical need. Arch Dermatol 2010;146:785-8.

2. Alenius GM, Stenberg B, Stenlund $H$, et al. Inflammatory joint manifestations are prevalent in psoriasis: prevalence study of joint and axial involvement in psoriatic patients, and evaluation of a psoriatic and arthritic questionnaire. J Rheumatol 2002;29:2577-82.

3. Gelfand JM, Gladman DD, Mease PJ, et al. Epidemiology of psoriatic arthritis in the population of the United States. J Am Acad Dermatol 2005;53:573.

4. Bhosle MJ, Kulkarni A, Feldman SR, et al. Quality of life in patients with psoriasis. Health Qual Life Outcomes 2006; 4:35.

5. Mallbris L, Ritchlin CT, Ståhle M. Metabolic disorders in patients with psoriasis and psoriatic arthritis. Curr Rheumatol Rep 2006;8:355-63.

6. Love TJ, Qureshi AA, Karlson EW, et al. Prevalence of the metabolic syndrome in psoriasis: results from the National Health and Nutrition Examination Survey, 2003-2006. Arch Dermatol 2011;147:419-24.

7. Gelfand JM, Neimann AL, Shin DB, et al. Risk of myocardial infarction in patients with psoriasis. JAMA 2006;296:1735-41.

8. Oureshi AA, Choi HK, Setty AR, et al. Psoriasis and the risk of diabetes and hypertension: a prospective study of US female nurses. Arch Dermatol 2009;145:379-82.

9. Schön MP, Boehncke WH. Psoriasis. N Engl J Med 2005;352:1899-912.

10. Hamminga EA, van der Lely AJ, Neumann HA, et al. Chronic inflammation in psoriasis and obesity: implications for therapy. Med Hypotheses 2006; 67:768-73.

11. Setty AR, Curhan G, Choi HK. Obesity, waist circumference, weight change, and the risk of psoriasis in women: Nurses' Health Study II. Arch Intern Med 2007:167:1670-5.

12. Tam LS, Tomlinson B, Chu TT, et al. Cardiovascular risk profile of patients with psoriatic arthritis compared to controls - the role of inflammation. Rheumatology (Oxford) 2008;47:718-23.

13. Bhole VM, Choi HK, Burns LC, et al. Differences in body mass index among individuals with PsA, psoriasis, RA and the general population. Rheumatology (Oxford) 2012; $51: 552-6$

14. Soltani-Arabshahi $\mathbf{R}$, Wong B, Feng BJ, et al. Obesity in early adulthood as a risk factor for psoriatic arthritis. Arch Dermatol 2010;146:721-6.

15. Blak BT, Thompson M. How does the health improvement network (thin) data on prevalence of chronic diseases compare with national figures? Value Health 2009:12:A253.

16. Seminara NM, Abuabara K, Shin DB, et al. Validity of The Health Improvement Network (THIN) for the study of psoriasis. Br J Dermatol 2011:164:602-9. 
17. Singh JA, Holmgren AR, Krug H, et al. Accuracy of the diagnoses of spondylarthritides in veterans affairs medical center databases. Arthritis Rheum 2007:57:648-55.

18. Rodríguez G, Soriano LC, Choi HK. Impact of diabetes against the future risk of developing gout. Ann Rheum Dis 2010;69:2090-4.

19. Toh S, Rodríguez LA, Hernández-Díaz S. Use of antidepressants and risk of lung cancer. Cancer Causes Control 2007;18:1055-64.

20. Lewis JD, Schinnar R, Bilker WB, et al. Validation studies of the health improvement network (THIN) database for pharmacoepidemiology research. Pharmacoepidemiol Drug Saf 2007;16:393-401.

21. Ruigómez A, Martín-Merino E, Rodríguez LA. Validation of ischemic cerebrovascular diagnoses in the health improvement network (THIN). Pharmacoepidemiol Drug Saf 2010;19:579-85.

22. Eder L, Law T, Chandran V, et al. Association between environmental factors and onset of psoriatic arthritis in patients with psoriasis. Arthritis Care Res (Hoboken) 2011;63:1091-7.

23. Pattison E, Harrison BJ, Griffiths CE, et al. Environmental risk factors for the development of psoriatic arthritis: results from a case-control study. Ann Rheum Dis 2008;67:672-6.

24. WHO. Obesity: preventing and managing the global epidemic. Report of a WHO Consultation. WHO Technical Report Series. Geneva: World Health Organization, 2000

25. Gisondi $\mathbf{P}$, Del Giglio M, Di Francesco V, et al. Weight loss improves the response of obese patients with moderate-to-severe chronic plaque psoriasis to low-dose cyclosporine therapy: a randomized, controlled, investigator-blinded clinical trial. Am J Clin Nutr 2008;88:1242-7.

26. de Menezes Ettinger JE, Azaro E, de Souza CA, et al. Remission of psoriasis after open gastric bypass. Obes Surg 2006;16:94-7.

27. Higa-Sansone G, Szomstein S, Soto F, et al. Psoriasis remission after laparoscopic Roux-en-Y gastric bypass for morbid obesity. Obes Surg 2004;14:1132-4.

28. Hossler EW, Maroon MS, Mowad CM. Gastric bypass surgery improves psoriasis. J Am Acad Dermatol 2011:65:198-200.

29. García Rodríguez LA, Varas-Lorenzo C, Maguire A, et al. Nonsteroidal antiinflammatory drugs and the risk of myocardial infarction in the general population. Circulation 2004;109:3000-6.

30. Martín-Merino E, García-Rodríguez LA, Massó-González EL, et al. Do oral antimuscarinic drugs carry an increased risk of acute urinary retention? J Urol 2009:182:1442-8

31. Hall GC. Validation of death and suicide recording on the THIN UK primary care database. Pharmacoepidemiol Drug Saf 2009;18:120-31.

32. Meal A, Leonardi-Bee J, Smith C, et al. Validation of THIN data for non-melanoma skin cancer. Qual Prim Care 2008;16:49-52.

33. Martín-Merino E, Ruigómez A, Johansson S, et al. Study of a cohort of patients newly diagnosed with depression in general practice: prevalence, incidence, comorbidity, and treatment patterns. Prim Care Companion J Clin Psychiatry 2010;12:PCC.08m00764. 